Laser Chem., 1999, Vol. 19, pp. 363-366

Reprints available directly from the publisher Photocopying permitted by license only
(C) 1999 OPA (Overseas Publishers Association) N.V.

Published by license under

the Harwood Academic Publishers imprint, part of The Gordon and Breach Publishing Group.

\title{
PICOSECOND TRANSIENT INFRARED SPECTROSCOPY OF 4-DIMETHYLAMINO- 4'-NITROSTILBENE IN THE FINGERPRINT REGION
}

\author{
HIROMI OKAMOTO* and MITSUO TASUMI ${ }^{\dagger}$ \\ Research Centre for Spectrochemistry and Department of Chemistry, \\ School of Science, The University of Tokyo, Bunkyo-ku, Tokyo 113, Japan
}

(Received 7 April 1997)

A picosecond infrared spectrometer covering nearly the whole fingerprint region has been constructed. The infrared absorption spectrum of electronically excited 4dimethylamino-4'-nitrostilbene, which has an intramolecular charge-transfer character, has been recorded. The observed infrared intensities are generally very strong (molar absorption coefficients as high as $3 \times 10^{3} \mathrm{~mol}^{-1} \mathrm{dm}^{3} \mathrm{~cm}^{-1}$ ). The infrared spectral pattern of the excited species is similar to that of the Raman spectrum. It is suggested that such features can be explained in terms of strong vibronic coupling in the observed excited species. Polarization measurements have also been performed.

Keywords: Time-resolved infrared spectroscopy; picosecond spectroscopy; intramolecular charge transfer; polarization measurements; vibronic coupling

Although ultrafast infrared spectroscopy is very important as a probe of ultrafast dynamics of molecular structures, its methodology is technically difficult especially in the fingerprint region [1], and is still in a developing stage. We have constructed a picosecond infrared spectrometer covering nearly the whole fingerprint region [2].

A picosecond infrared light source tunable in the fingerprint region was obtained by difference frequency mixing of a Nd:YLF fundamental output $(1.053 \mu \mathrm{m})$ and a tunable near-infrared light $(1.15-1.3 \mu \mathrm{m})$,

\footnotetext{
*Corresponding author.

${ }^{\dagger}$ Present address: Department of Chemistry, Faculty of Science, Saitama University, Urawa, Saitama 338, Japan.
} 
which was generated by difference frequency mixing of the Nd:YLF fundamental and a tunable visible light $(545-578 \mathrm{~nm})$ from a dye laser. The infrared absorption spectrum of electronically excited 4-dimethylamino-4'-nitrostilbene (DNS), which is considered to have an intramolecular charge-transfer character, has been recorded. In addition to negative bands due to the bleach of the ground-state molecules, several strong bands due to the excited-state molecules were observed [2]. The observed infrared bands and their tentative assignments are summarized in Table I, together with the Raman bands of the same species [3]. The molar absorption coefficients of the observed prominent infrared bands were estimated to be as high as $3 \times 10^{3} \mathrm{~mol}^{-1} \mathrm{dm}^{3} \mathrm{~cm}^{-1}$. The observed infrared spectral pattern of the excited species was found to be similar to that of the Raman spectrum in some points. We consider that this similarity as well as the very large absorption coefficients can be explained on the basis of vibronic interaction as mentioned below.

If the electronic state of the observed species $[e]$ is expanded in terms of unperturbed electronic states $\left.\left\{\mid s_{0}\right]\right\}$ at the equilibrium nuclear geometry (Herzberg-Teller expansion), we have

$$
\begin{aligned}
& \left.\left.\left.\left.\mid e] \mid 0) \approx \mid e_{0}\right] \mid 0\right)+\sum_{s \neq e}\left(h_{Q}\right)_{e s} a_{e s}^{0} \mid s_{0}\right] \mid 1\right) \\
& \left.\left.\left.\left.\left.\left.\mid e] \mid 1) \approx \mid e_{0}\right] \mid 1\right)+\sum_{s \neq e}\left(h_{Q}\right)_{e s}\left\{a_{e s}^{0} \mid s_{0}\right] \mid 0\right)+a_{e s}^{1} \mid s_{0}\right] \mid 2\right)\right\}
\end{aligned}
$$

TABLE I Vibrational frequencies (in $\mathrm{cm}^{-1}$ ) of the excited DNS in a benzonitrile solution

\begin{tabular}{lcccc}
\hline Infrared $^{\text {ger; }}$ & Intensity $^{\mathrm{a}}$ & Raman $^{\mathrm{b}}$ & Intensity $^{\mathrm{a}}$ & Assignment $^{\mathrm{c}}$ \\
\hline 1622 & $\mathrm{~m}$ & $1626^{\mathrm{d}}$ & $\mathrm{m}^{\mathrm{d}}$ & olefinic C=C \\
1574 & $\mathrm{~s}$ & 1582 & $\mathrm{~s}$ & ring str. \\
1543 & $\mathrm{w}$ & 1475 & $\mathrm{w}$ & \\
& & $\approx 1375$ & $\mathrm{w}$ & \\
1383 & $\mathrm{w}$ & 1280 & $\mathrm{~s}$ & $\mathrm{NO}_{2}$ sym.str. \\
1271 & $\mathrm{~s}$ & 1188 & $\mathrm{~s}$ & \\
1191 & $\mathrm{~m}$ & 1165 & $\mathrm{sh}$ & \\
& & 1052 & $\mathrm{~m}$ & ring C $-\mathrm{N}$ str. \\
1053 & $\mathrm{~s}$ & 988 & $\mathrm{vw}$ & \\
963 & $\mathrm{w}$ & & & \\
\hline
\end{tabular}

a s, strong; m, medium; w, weak; vw, very weak; sh, shoulder.

b Ref. [3].

c Tentative assignment.

d Raman shift and intensity in an acetonitrile solution. 
where $Q$ represents a nuclear coordinate which induces the vibronic interaction, and $\left(h_{Q}\right)_{e s}$ a vibronic coupling matrix element for that mode and $\mid v)$ and $a_{e s}^{v}$ denote, respectively, the vibrational state and a coefficient dependent on the vibrational quantum number $v$. Infrared transition moment $\mu_{Q}$ for the $1 \leftarrow 0$ vibrational transition of the $Q$ mode in the $e$ state is given by the following equation.

$$
\mu_{Q} \sim \sum_{s \neq e}\left(h_{Q}\right)_{e s} a_{e s}^{0}\left[e_{0}|\mu| s_{0}\right]
$$

This means that the vibrational transition borrows the intensity from that of the strong electronic transition. On the other hand, the $C$ term of Raman tensor [4] for the $1 \leftarrow 0$ vibrational transition of the $Q$ mode is given by the following equation.

$$
C_{Q} \sim \sum_{s \neq e}\left(h_{Q}\right)_{e s}\left[e_{0}|\mu| t_{0}\right]\left[t_{0}|\mu| s_{0}\right] \sum_{v} a_{e s}^{0}\left\{(0 \mid v)^{2}+(1 \mid v)^{2}\right\} /\left(E_{t v}-h \nu_{0}+i \Gamma\right)
$$

where $\left.\mid t_{0}\right]$ denotes the resonant electronic state and $E_{t v}$ is the energy difference between the initial and virtual vibronic levels.

In a charge-transfer system, electron-density distribution alters seriously when the nuclear configuration changes. It corresponds to the existence of a strong vibronic interaction, or in other words, large $\left(h_{Q}\right)_{e s}$ and $a_{e s}^{v}$ values. Then, it gives a large $\mu_{Q}$ which explains the very strong infrared absorption. At the same time, a large $C_{Q}$ is also expected under strong vibronic interaction. If the $C_{Q}$ has a significant contribution to the whole Raman tensor, a strong Raman band due to the $Q$ mode may be observed. As a result, vibrational modes which induce strong vibronic interaction in the $e$ state may give strong bands in both the infrared and Raman spectra. This situation is possibly common to the vibrational spectra of other electronically excited and charged species with proper symmetries.

Measurements of polarization dependence were performed for several prominent bands. It provides valuable information on vibrational assignments of the infrared bands and structural changes upon electronic excitation. The observed polarization characteristics are consistent with the tentative band assignments. 


\section{References}

[1] Hamm, P., Zurek, M., Mäntele, W., Meyer, M., Scheer, H. and Zinth, W. (1995). Proc. Natl. Acad. Sci. USA, 92, 1826.

[2] Okamoto, H. and Tasumi, M. (1996). Chem. Phys. Lett., 256, 502.

[3] Nakabayashi, T., Okamoto, H. and Tasumi, M. (1995). J. Raman Spectrosc., 26, 841.

[4] Albrecht, A. C. (1961). J. Chem. Phys., 34, 1476. 